\title{
Influencia del envío de mensajes de texto al teléfono móvil en el control glucémico de la diabetes tipo 2 \\ Influence of sending messages to the mobile phone in the glycemic control of diabetes type 2
}

Alba García-Violaa

aDepartamento de Enfermería, Fisioterapia y Medicina, Facultad de Ciencias de la Salud, Universidad de Almeria, España

\section{Resumen}

Introducción: La prevalencia de diabetes mellitus tipo 2 en adultos se ha incrementado significativamente en la última década, considerándose un problema de salud pública. Los nuevos avances tecnológicos pueden resultar de gran utilidad en el manejo de la enfermedad. Objetivos: Evaluar la evidencia científica disponible sobre el aviso mediante mensajes de texto al teléfono móvil de los pacientes diabéticos tipo 2, y determinar cómo influye en el control glucémico, en la adhesión al tratamiento, así como en los niveles de hemoglobina glicosilada (HbA1c). Metodología: Se realizó una revisión bibliográfica en la que se encontraron 12 artículos, los cuales, cumplían con los criterios de inclusión. Se consultaron las siguientes bases de datos: Pubmed, Scopus, Lilacs, Dialnet Plus y Cuiden Plus. Resultados: De acuerdo con los textos revisados, el uso de las nuevas tecnologías se asocia con una disminución de los niveles de $\mathrm{HbA} 1 \mathrm{c}$, una mejora de los controles glucémicos, una mayor adquisición de conocimientos sobre la enfermedad y estilo de vida, y una mayor adherencia al tratamiento terapéutico. Además, los pacientes identificaron estas intervenciones como una herramienta muy útil y motivadora. Conclusión: El envío de mensajes de texto a los pacientes con diabetes tipo 2 tiene un papel muy importante en el cuidado y manejo de la enfermedad.

Palabras Clave: Medios de Comunicación; Diabetes Mellitus Tipo 2; Teléfono inteligente; Mensaje de Texto; Autocontrol; Hemoglobina glicosilada.

\begin{abstract}
Introduction: The prevalence of type 2 diabetes mellitus in adults has increased significantly in the last decade, being considered a public health problem. New technological advances can be very useful in the management of the disease. Objectives: To evaluate the available evidence about the warning by means of text messages to the mobile phone of the diabetic patients Type 2 and to determine how it influences in the glycemic control, in the adherence to the treatment, as well as in the levels of glycosylated hemoglobin. Methodology: A literature review was conducted where 12 articles, which met the previous established inclusion criteria, were found. The following databases were consulted for the literature review: Pubmed, Scopus, Lilacs, Dialnet Plus and Cuiden Plus. Results: According to the revised texts, the use of new technologies is associated with a decrease in $\mathrm{HbA} 1 \mathrm{c}$ levels, improvement of glycemic controls, a greater acquisition of knowledge about the disease and lifestyle, and greater adherence to therapeutic treatment. In addition, patients identified these interventions as a very useful and motivating tool. Conclusions: Sending text messages to patients with type 2 diabetes plays a very important role in the care and management of the disease.
\end{abstract}

Key Words: Communications Medium; Diabetes Mellitus Type 2; Smartphone; Text Messaging; Selfmanagement; Glycosylated hemoglobin. 


\section{Introducción}

a Diabetes Mellitus es una de las enfermedades que generan un mayor impacto sociosanitario en la actualidad. Desde 1980 la prevalencia mundial de personas diabéticas ha ascendido del $4,7 \%$ al $8,5 \%$ en la población adulta (Organización Mundial de la Salud, 2014; Organización Mundial de la Salud, 2016). Según predicciones de la OMS, en el año 2030, la séptima causa de mortalidad mundial será la diabetes (Mathers \& Loncar, 2006).

Cabe destacar que el $90-95 \%$ de los casos de personas diabéticas corresponden a Diabetes Mellitus Tipo 2 (DM2) (Porth, Gaspard, \& Noble, 2011). Estos datos se deben en gran parte a la existencia de factores de riesgo tales como: la obesidad o el sobrepeso, los estilos de vida sedentarios, el consumo de tabaco y de alcohol, las dietas hipercalóricas... entre otras más (Llorente Columbié, Miguel-Soca, Rivas Vázquez, \& Borrego Chi, 2016). No obstante, la modificación de estos factores de riesgo puede prevenir la progresión de la enfermedad, la aparición de complicaciones y la muerte precoz (Wu, Ding, Tanaka, \& Zhang, 2014).

Actualmente, la DM2 se sitúa como uno de los problemas más comprometidos del ámbito sanitario debido, en gran parte, a las complicaciones que provoca la presencia de esta enfermedad y el alto riesgo de ingresos hospitalarios. Esto es consecuencia de una mala adhesión al tratamiento farmacológico y un mal control de la enfermedad, resultando difícil lograr un manejo eficaz (Breitscheidel, Stamenitis, Dippel, \& Schöffski, 2010; Lee, Greenfield, \& Pappas, 2018).

Enfermería juega un papel primordial en la educación de los pacientes con DM2, se encarga de ofrecer apoyo, educación sanitaria y orientarles sobre las modificaciones en el estilo de vida, con el fin de reducir la progresión de la enfermedad, las complicaciones asociadas y mejorar la calidad de vida del paciente diabético (Thiessen, 2017). Es necesario que el paciente adquiera conocimientos sobre la realización de ejercicio físico, la dieta a seguir, el ajuste del tratamiento, los controles glucémicos, el manejo ante hipoglucemias e hiperglucemias, la administración del tratamiento farmacológico, así como informarles sobre las complicaciones que derivan de su enfermedad y de los programas de prevención existentes (Ariza Copado et al., 2011; Del Prato et al., 2005). A pesar de las recomendaciones dadas, los profesionales sanitarios se encuentran con la falta de continuidad en el tratamiento, dado que más del $60 \%$ de los pacientes no cumple los objetivos propuestos. Es por ello que resulta necesario implementar otras estrategias alternativas que permitan lograr mejores resultados (Chen, Magliano, \& Zimmet, 2011; Del Prato et al., 2005; Lawrenson, Joshy, Eerens, \& Johnstone, 2010).

Según la OMS, el uso de los dispositivos móviles resulta beneficioso para lograr los objetivos propuestos en salud (Organización Mundial de la Salud, 2016). La tecnología móvil ha ido extendiéndose por todo el mundo en los últimos años, resultando de gran utilidad en el control y manejo de las enfermedades crónicas, entre ellas la DM2 (Chan \& Kaufman, 2010; Mechael et al., 2010). Su uso se extiende por todas las clases sociales y edades, suponiendo una gran ventaja el acceso universal a estos dispositivos, ya que cualquier persona puede usarlos independientemente de la zona geográfica en la que se sitúe (Benito Badorrey, 2015). Por todo ello, su uso está incluyéndose en el desarrollo de aplicaciones móviles y de programas específicos que envían mensajes de texto personalizados y de forma automática al teléfono móvil de los pacientes diabéticos, ayudándoles y animándoles a participar en el control y en el automanejo de su enfermedad. Se trata de una oportunidad única para mejorar la adherencia a sus regímenes terapéuticos. (Arambepola et al., 2016; Benito Badorrey, 2015; Liang et al., 2011; Nundy et al., 2014, Saffari, Ghanizadeh, \& Koenig, 2014).

\section{Objetivos}

- Identificar la evidencia científica disponible sobre el aviso mediante notificaciones/mensajes de texto al teléfono móvil de los pacientes diabéticos tipo 2 y comprobar la efectividad de esta intervención en el control glucémico de su enfermedad.

- Determinar cómo influye el empleo de las diferentes tecnologías de la información en la adhesión al tratamiento de los pacientes diabéticos tipo 2 y en los niveles de hemoglobina glicosilada en sangre.

\section{Metodología}

Se trata de una revisión bibliográfica de lo publicado en las bases de datos de Ciencias de la Salud. La búsqueda se llevó a cabo consultando los Descriptores de Ciencias de la Salud (DeCS) con las palabras clave: Medios de Comunicación (Communications Media), Diabetes Mellitus (Diabetes Mellitus), Telecomunicaciones (Telecommunications), Teléfono Inteligente (Smartphone), Mensaje de Texto (Text Messaging) y Autocontrol (Selfmanagement). También se consultaron los siguientes Medical Subject Headings (MeSH): Diabetes Mellitus Type 2, Cell Phone, Text Messaging y Self-management. Las bases de datos consultadas durante la búsqueda han 
sido: Pubmed, Scopus, Lilacs, Dialnet Plus y Cuiden Plus.

Se establecieron como criterios de inclusión todos aquellos artículos que estuviesen relacionados con el empleo de diferentes tecnologías de la información (teléfonos móviles, notificaciones, mensajes de texto... entre otras) y los pacientes diabéticos tipo 2, que hayan sido publicados entre el año 2008 hasta la actualidad y que estuviesen escritos en cualquier idioma, siempre y cuando, incluyesen los criterios mencionados anteriormente. Se excluyeron los artículos que hacían referencia a otros tipos de diabetes y, además, aquellos artículos de escasa evidencia científica.

La revisión se llevó a cabo desde el mes de diciembre del 2018 a febrero del 2019. De las diferentes bases de datos consultadas se seleccionaron un total de 12 artículos publicados en los últimos años. La estrategia de búsqueda se llevó a cabo a través de la combinación de las diferentes palabras clave y el uso de los operadores booleanos AND, NOT y OR.

A continuación, en la Figura 1 se representan los resultados de la búsqueda bibliográfica mediante el diagrama de flujo PRISMA.

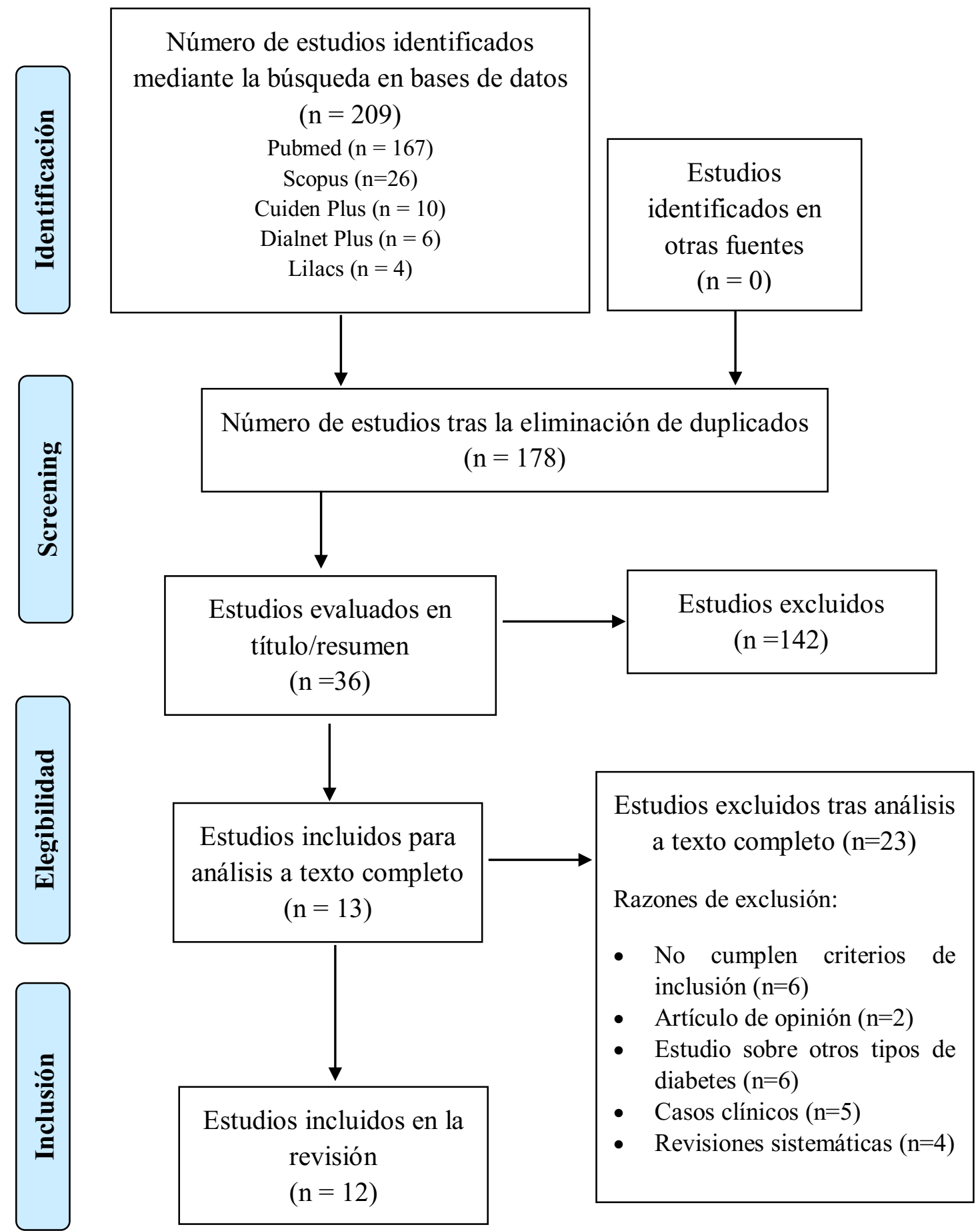

Figura 1. Diagrama de flujo PRISMA 


\section{Resultados}

Para la elaboración de los resultados se analizaron 12 estudios, los cuales, cumplían con los criterios de inclusión previamente establecidos. El diseño de los diferentes estudios revisados fue:

- Ensayos aleatorios controlados (ECA) (Bergner, Nelson, Rothman, \& Mayberry, 2017; Capozza et al., 2015; Dobson et al., 2018; Fortmann et al., 2017; Goodarzi, Ebrahimzadeh, Rabi, Saedipoor, \& Jafarabadi, 2012; Hussein, Hasan, \& Jaradat, 2011; Wargny et al., 2018). - Estudios cuasi-experimentales (Adikusuma \& Qiyaam, 2017; Nelson, Mulvaney, Gebretsadik, Johnson, \& Osborn, 2016).

- Ensayo experimental no aleatorio prospectivo (Bassam Bin, Al Fares, Jabbari, El Dali, \& Al Orifi, 2015).

- Entrevistas semiestructuradas. Análisis fenomenológico (Lee, Greenfield, \& Pappas, 2018).

- Grupos focales (Chen, Yu, Li, Zhan, \& Yan, 2018).

\section{Características de la población de estudio}

De las 12 investigaciones revisadas, en seis de ellas la población de estudio fueron exclusivamente personas adultas diagnosticadas de DM2 (Adikusuma \& Qiyaam, 2017; Bergner et al., 2017; Chen et al., 2018; Goodarzi et al., 2012; Nelson et al., 2016; Wargny et al., 2018). En otro estudio contaban con pacientes diagnosticados de DM2 sin presencia de complicaciones derivadas de la propia enfermedad (Bassam Bin et al., 2015). En cuatro investigaciones se incluyeron a diabéticos tipo 2 que tenían un mal control de su enfermedad (Capozza et al., 2015; Dobson et al., 2018; Fortmann et al., 2017; Hussein et al., 2011) y alto riesgo de complicaciones derivadas de la misma (Capozza et al., 2015). Otro de los estudios tuvo en cuenta a pacientes que padeciesen alguna enfermedad crónica aparte de la DM2 (Lee et al., 2018).

\section{Resultados obtenidos}

Se analizaron diferentes intervenciones que empleaban nuevas tecnologías de la información, las cuales, envían mensajes de texto personalizados al teléfono móvil de los pacientes. En estos mensajes se incluía información sobre el autocuidado, la dieta, el ejercicio, el manejo terapéutico, el control de los niveles de glucosa en sangre, entre otros. Los grupos controles de los estudios analizados recibieron atención habitual por su médico y enfermera de referencia.
Se observó que el uso de las nuevas tecnologías se asocia con una reducción y una mejora de los niveles de $\mathrm{HbA} 1 \mathrm{C}$ desde el inicio de las intervenciones. Cabe destacar que la duración de estas intervenciones no fue del mismo periodo de tiempo, éstas han oscilado entre los 3 y los 9 meses de duración (Bassam Bin et al., 2015; Capozza et al., 2015; Dobson et al., 2018; Fortmann et al., 2017, Goodarzi et al., 2012; Hussein et al., 2011; Wargny et al., 2018). Asimismo, se evidenció una reducción de los niveles de LDL, colesterol y microalbúmina en sangre (Goodarzi et al., 2012). Sin embargo, en uno de los estudios se analizó que el envío de mensajes de texto no influía en los niveles de $\mathrm{HbA} 1 \mathrm{C}$ ya que no se observaron diferencias significativas, aunque sí logró conseguir una mayor adhesión terapéutica en los pacientes (Nelson et al., 2016).

También se observó que los pacientes que recibían mensajes de texto y notificaciones al teléfono móvil adquirían mayores conocimientos sobre su enfermedad, la dieta, el ejercicio, el tratamiento terapéutico y la importancia de controlar los niveles de glucemia. Además, lograban un manejo eficaz de su enfermedad (Bassam Bin et al., 2015; Capozza et al., 2015; Goodarzi et al., 2012) y una mayor adherencia al tratamiento terapéutico que aquellos pacientes que recibían la atención habitual (Adikusuma \& Qiyaam, 2017; Bassam Bin et al., 2015; Nelson et al., 2016). Asimismo, se consiguió un mejor control glucémico (Fortmann et al., 2017; Wargny et al., 2018), reduciéndose los niveles de glucemia en ayunas (Adikusuma \& Qiyaam, 2017; Bassam Bin et al., 2015) y los niveles de glucosa post-pandrial (Adikusuma \& Qiyaam, 2017). No obstante, en uno de los estudios se observó que no hubo diferencias significativas en cuanto a la dieta, el ejercicio, los niveles de glucosa en sangre y en la conducta de dejar de fumar (Dobson et al., 2018).

En cuanto a la satisfacción y actitud de los pacientes frente al empleo de estas nuevas tecnologías, las identificaron como unas herramientas muy útiles y motivadoras. Les ayudaban a mejorar su calidad de vida, permitiéndoles ser más independientes, recordándoles cuando tomar la medicación, dándoles consejos sobre su estilo de vida y permitiéndoles tener un mayor control sobre su propia salud, todo ello, a través de los consejos y recomendaciones que les llegaban a su teléfono móvil (Bergner et al., 2017; Chen et al., 2018; Lee et al., 2018).

En la tabla 1 se pueden ver resumidos los artículos analizados. 
Tabla 1. Resumen artículos.

\begin{tabular}{|c|c|c|c|}
\hline Estudio & Tecnología empleada & Muestra & Resultados \\
\hline Adikusuma \& Qiyaam, (2017) & $\begin{array}{l}\text { Envío de mensajes } \\
\text { personalizados al teléfono } \\
\text { móvil. }\end{array}$ & $N=50$ & $\begin{array}{l}\text { Mayor control de los niveles de } \\
\text { glucemia y adhesión terapéutica. }\end{array}$ \\
\hline $\begin{array}{l}\text { Bassam Bin, Al Fares, } \\
\text { Jabbari, El Dali \& Al Orifi, } \\
(2015)\end{array}$ & $\begin{array}{l}\text { Envío de mensajes de } \\
\text { texto al teléfono móvil } \\
\text { más llamadas telefónicas } \\
\text { para asegurarse de que lo } \\
\text { habían recibido. }\end{array}$ & $N=100$ & $\begin{array}{l}\text { Aumento del conocimiento de la } \\
\text { enfermedad y mejor control de } \\
\text { los niveles de glucosa en ayunas. }\end{array}$ \\
\hline $\begin{array}{l}\text { Bergner, Nelson, Rothman \& } \\
\text { Mayberry, (2017) }\end{array}$ & $\begin{array}{l}\text { Uso de la aplicación } \\
\text { REACH más entrevista } \\
\text { semiestructurada por } \\
\text { teléfono para comprobar } \\
\text { la eficacia o no de la } \\
\text { aplicación. }\end{array}$ & $N=55$ & $\begin{array}{l}\text { Programa útil y motivador, que } \\
\text { permite una mejor adhesión } \\
\text { terapéutica y estilo de vida. }\end{array}$ \\
\hline Capozza et al., (2015) & $\begin{array}{l}\text { Atención estándar Vs. } \\
\text { Atención estándar más } \\
\text { programa que envía } \\
\text { mensajes de texto al } \\
\text { teléfono móvil (Care4Life). }\end{array}$ & $N=93$ & $\begin{array}{l}\text { Disminución de los niveles de } \\
\text { HbA1c en ambos grupos. Sin } \\
\text { embargo, los pacientes del } \\
\text { grupo experimental mejoraron } \\
\text { su calidad de vida y adquirieron } \\
\text { nuevos conocimientos. }\end{array}$ \\
\hline $\begin{array}{l}\text { Chen, Yu, Li, Zhan \& Yan, } \\
\text { (2018) }\end{array}$ & $\begin{array}{l}\text { Perspectivas sobre el envío } \\
\text { de mensajes de texto al } \\
\text { teléfono móvil }\end{array}$ & $\mathrm{N}=9$ & $\begin{array}{l}\text { Los participantes mostraron una } \\
\text { actitud positiva ante esta nueva } \\
\text { tecnología, ya que lograban una } \\
\text { mejor adhesión al tratamiento y } \\
\text { mayores conocimientos. }\end{array}$ \\
\hline Dobson et al., (2018) & $\begin{array}{l}\text { Atención estándar } \\
\text { Vs. } \\
\text { Envío de mensajes de } \\
\text { texto personalizados al } \\
\text { teléfono móvil (SMS4BG). }\end{array}$ & $\mathrm{N}=366$ & $\begin{array}{l}\text { Reducción de los niveles de } \\
\text { HbA1c y mejor control de la } \\
\text { enfermedad. }\end{array}$ \\
\hline Fortmann et al., (2017) & $\begin{array}{l}\text { Envío de mensajes de } \\
\text { texto al móvil a través de la } \\
\text { aplicación Dulce Digital } \\
\text { Vs. } \\
\text { Atención estándar. }\end{array}$ & $\mathrm{N}=126$ & $\begin{array}{l}\text { Reducción de los niveles de } \\
\text { HbA1C y mejor control de los } \\
\text { valores de glucemia en sangre. }\end{array}$ \\
\hline $\begin{array}{l}\text { Goodarzi, Ebrahimzadeh, } \\
\text { Rabi, Saedipoor \& Jafarabadi, } \\
\text { (2012) }\end{array}$ & $\begin{array}{l}\text { Envío de mensajes } \\
\text { personalizados al teléfono } \\
\text { móvil. }\end{array}$ & $N=81$ & $\begin{array}{l}\text { Se observó una mejora } \\
\text { significativa en los niveles } \\
\text { de HbA1c, LDL, colesterol y } \\
\text { microalbúmina. Así como en los } \\
\text { conocimientos y el autocontrol de } \\
\text { la enfermedad. }\end{array}$ \\
\hline
\end{tabular}


Tabla 1. Continuación.

\begin{tabular}{|l|l|l|l|}
\hline \multicolumn{1}{|c|}{ Estudio } & \multicolumn{1}{|c|}{ Tecnología empleada } & \multicolumn{1}{c|}{ Muestra } & \multicolumn{1}{c|}{ Resultados } \\
\hline $\begin{array}{l}\text { Hussein, Hasan \& Jaradat, } \\
\text { (2011) }\end{array}$ & $\begin{array}{l}\text { Envío de mensajes de texto } \\
\text { personalizados al móvil } \\
\text { a través de la aplicación } \\
\text { (SMS-DM) más asistir a la } \\
\text { consulta habitual. }\end{array}$ & $\mathrm{N}=34$ & $\begin{array}{l}\text { Reducción de los niveles de } \\
\text { HbA1C }\end{array}$ \\
\hline $\begin{array}{l}\text { Lee, Greenfield \& Pappas, } \\
\text { (2018) }\end{array}$ & $\begin{array}{l}\text { Percepciones de los } \\
\text { pacientes sobre el uso de } \\
\text { la telesalud. }\end{array}$ & $\mathrm{N}=10$ & $\begin{array}{l}\text { Se apoya el uso de la telesalud. } \\
\text { Todos los pacientes afirmaban } \\
\text { que estaban muy satisfechos, } \\
\text { mejorando su calidad de vida y } \\
\text { permitiéndoles tener más control } \\
\text { sobre su salud. }\end{array}$ \\
\hline $\begin{array}{l}\text { Nelson, Mulvaney, } \\
\text { Gebretsadik, Johnson \& } \\
\text { Osborn, } \\
\text { (2016) }\end{array}$ & $\begin{array}{l}\text { Envío de mensajes de } \\
\text { texto al teléfono móvil más } \\
\text { lamada telefónica semanal } \\
\text { Vs. } \\
\text { Atención habitual. }\end{array}$ & $\mathrm{N}=80$ & $\begin{array}{l}\text { No hubo diferencias en los } \\
\text { niveles de HbA1C, pero tuvo un } \\
\text { impacto positivo en la adherencia } \\
\text { terapéutica. }\end{array}$ \\
\hline Wargny et al., (2018) & $\begin{array}{l}\text { Envío de mensajes } \\
\text { personalizados al teléfono } \\
\text { móvil. }\end{array}$ & $\mathrm{N}=186$ & $\begin{array}{l}\text { Disminución de los niveles de } \\
\text { HbA1c y mejor control glucémico. }\end{array}$ \\
\hline
\end{tabular}

\section{Discusión}

Con el fin de crear un documento de evidencia para comprobar la eficacia del envío de mensajes de texto en pacientes con DM2, se ha realizado una revisión bibliográfica con el objetivo de generar resultados precisos y conclusiones fiables. En esta revisión se evidenció que el envío de mensajes de texto a través del teléfono móvil se traduce en una reducción de los niveles de $\mathrm{HbA} 1 \mathrm{c}$. Estos datos se deben principalmente al empleo de los mensajes de texto, ya que los siete estudios cuyos resultados concluyen ese dato, al término de los mismos, han utilizado esta tecnología como intervención.

En tres estudios se utilizó la realización de llamadas telefónicas por parte del personal sanitario como método de apoyo al envío de los mensajes de texto, y así, asegurarse de que los recibían correctamente y hacer un seguimiento semanal de la enfermedad (Bassam Bin et al., 2015, Bergner et al., 2017; Nelson et al., 2016,). E resto de los estudios se basaron exclusivamente en el envío de mensajes de texto individuales 0 a través de una aplicación móvil.

Haciendo referencia a la adquisición de conocimientos y al cambio en el estilo de vida de los pacientes en cuanto a dieta, ejercicio y niveles glucémicos, tres estudios demostraron que los pacientes adoptaban mejores conductas (Bassam Bin et al., 2015; Capozza et al., 2015; Goodarzi et al., 2012), en cambio, Dobson et al. (2018) no detectaron diferencias significativas en esos parámetros.

Una limitación del presente trabajo ha sido el escaso número de artículos científicos incluidos en la presente revisión, ya que en el momento de su realización no se encontró mayor evidencia de calidad acerca del tema. Además, la gran parte de investigaciones originales suele publicarse en formato de artículo, pero en otras ocasiones, los autores optan por publicar sus hallazgos en otros formatos, tales como artículos de opinión o casos clínicos, lo que puede suponer que los resultados obtenidos en esta revisión no muestren el total de las investigaciones llevadas a cabo acerca del tema.

Como recomendación para futuras investigaciones se propone que los próximos estudios se deberían centrar en el impacto que tienen estas intervenciones en otras áreas del manejo de la diabetes, tales como la calidad de vida tras la aplicación de las intervenciones. Además, también se debería analizar cómo influyen estas intervenciones en otros parámetros sanguíneos donde se han observado que tienen un impacto positivo, pero los estudios son escasos. 


\section{Conclusiones}

El envío de mensajes de texto personalizados a los pacientes con DM2 tiene un papel muy importante en el cuidado y en el manejo de la enfermedad. La efectividad de estas intervenciones se traduce principalmente en una disminución de los niveles de $\mathrm{HbA} 1 \mathrm{c}$, un aumento de los conocimientos y una mejor adhesión terapéutica y manejo de la enfermedad, en comparación con los pacientes que recibían una atención habitual por su médico o enfermera de referencia.

\section{Referencias Bibliográficas}

Adikusuma, W., \& Qiyaam, N.(2017). Theeffect ofeducation through short message service (SMS) Messages on diabetic patients adherence. Scientia Pharmaceutica, 85(2), 23. doi: 10.3390/scipharm85020023

Arambepola, C., Ricci-Cabello, I., Manikavasagam, P., Roberts, N., French, D., \& Farmer, A. (2016). The impact of automated brief messages promoting lifestyle changes delivered via mobile devices to people with Type 2 Diabetes: A systematic literature review and meta-analysis of controlled trials. Journal of Medical Internet Research, 18(4), e86. doi: 10.2196/jmir.5425

Ariza Copado, C., Gavara Palomar, V., Munoz Ureña, A., Aguera Mengual, F., Soto Martínez, M., \& Lorca Serralta, J. (2011). Mejora en el control de los diabéticos tipo 2 tras una intervención conjunta: educación diabetológica y ejercicio físico. Atención Primaria, 43(8), 398-406.

Bassam Bin, A., Al Fares, A., Jabbari, M., El Dali, A., \& Al Orifi, F. (2015). Effect of mobile phone short text messages on glycemic control in Type 2 Diabetes. International Journal of Endocrinology and Metabolism, 13(1). doi: 10.5812/ijem.18791

Benito Badorrey, B. (2015). Diabetes mellitus en los dispositivos móviles. Diabetes Práctica, 6(2), 49-96.

Bergner, E., Nelson, L., Rothman, R., \& Mayberry, L. (2017). Text messaging may engage and benefit adults with Type 2 Diabetes regardless of health literacy status. HLRP: Health Literacy Research and Practice, 1(4). doi: 10.3928/24748307-20170906-01

Breitscheidel, L., Stamenitis, S., Dippel, F., \& Schöffski, O. (2010). Economic impact of compliance to treatment with antidiabetes medication in type 2 diabetes mellitus: a review paper. Journal of Medical Economics, 13(1), 8-15.

Capozza, K., Woolsey, S., Georgsson, M., Black, J., Bello,
N., Lence, C., ... North, C. (2015). Going mobile with diabetes support: a randomized study of a text messagebased personalized behavioral intervention for Type 2 Diabetes self-care. Diabetes Spectrum, 28(2), 83-91. doi: $10.2337 /$ diaspect.28.2.83

Chan, C., \& Kaufman, D. (2010). A technology selection framework for supporting delivery of patient-oriented health interventions in developing countries. Journal of Biomedical Informatics, 43, 300-306. doi: 10.1016/j. jbi.2009.09.006

Chen, L., Magliano, D., \& Zimmet, P. (2011). The worldwide epidemiology of type 2 diabetes mellitus-present and future perspectives. Nature Reviews Endocrinology, 8(4), 228-236.

Chen, X., Yu, S., Li, C., Zhan, X., \& Yan, W. (2018). Text message-based intervention to improve treatment adherence among rural patients with type 2 diabetes mellitus: a qualitative study. Public Health, 163, 46-53.

Del Prato, S., Felton, A., Munro, N., Nesto, R., Zimmet, P., \& Zinman, B. (2005). Improving glucose management: Ten steps to get more patients with type 2 diabetes to glycaemic goal. International Journal of Clinical Practice, 59(11), 1345-1355.

Dobson, R., Whittaker, R., Jiang, Y., Maddison, R., Shepherd, M., McNamara, C., ... Murphy, R. (2018). Effectiveness of text message based, diabetes self management support programme (SMS4BG): Two arm, parallel randomized controlled trial. BMJ, 361. doi: 10.1136/bmj.k1959

Fortmann, A. L., Gallo, L. C., Garcia, M. I., Taleb, M., Euyoque, J. A., Clark, T., ... Philis-Tsimikas, A. (2017). Dulce digital: An mHealth SMS-based intervention improves glycemic control in hispanics with Type 2 Diabetes. Diabetes Care, 40(10), 1349-1355. doi: $10.2337 / \mathrm{dc} 17-0230$

Goodarzi, M., Ebrahimzadeh, I., Rabi, A., Saedipoor, B., \& Jafarabadi, M. (2012). Impact of distance education via mobile phone text messaging on knowledge, attitude, practice and self efficacy of patients with type 2 diabetes mellitus in Iran. Journal of Diabetes \& Metabolic Disorders, 11(1), 10. doi: 10.1186/2251-6581-11-10

Hoyos Duque, T., Arteaga Henao, M., \& Muñoz Cardona, M. (2011). Factores de no adherencia al tratamiento en personas con Diabetes Mellitus tipo 2 en el domicilio. Revista de Investigación y Educación en Enfermería, 
29(2), 194-203.

Hussein, W., Hasan, K., \& Jaradat, A. (2011). Effectiveness of mobile phone short message service on diabetes mellitus management; the SMS-DM study. Diabetes Research and Clinical Practice, 94(1), e24-e26. doi: 10.1016/j.diabres.2011.07.025

Lawrenson, R., Joshy, G., Eerens, Y., \& Johnstone, W. (2010). How do newly diagnosed patients with type 2 diabetes in the Waikato get their diabetes education? Journal of Primary Health Care, 2(4), 303.

Lee, P., Greenfield, G., \& Pappas, Y. (2018). Patients' perception of using telehealth for type 2 diabetes management: a phenomenological study. BMC Health Services Research, 18(1).

Lee, P., Greenfield, G., \& Pappas, Y. (2018). The impact of telehealth remote patient monitoring on glycemic control in type 2 diabetes: a systematic review and meta-analysis of systematic reviews of randomised controlled trials. BMC Health Services Research, 18(1).

Liang, X., Wang, Q., Yang, X., Cao, J., Chen, J., Mo, X., ... Gu, D. (2011). Effect of mobile phone intervention for diabetes on glycaemic control: A meta-analysis. Diabetic Medicine, 28(4), 455-463. doi: 10.1111/j.14645491.2010.03180.x

Llorente Columbié, Y., Miguel-Soca, P., Rivas Vázquez, D., \& Borrego Chi, Y. (2016). Factores de riesgo asociados con la aparición de diabetes mellitus tipo 2 en personas adultas. Revista Cubana de Endocrinología, 27(2), 123-133.

Mathers, C., \& Loncar, D. (2006). Projections of global mortality and burden of disease from 2002 to 2030. Plos Medicine, 3(11).

Mechael, P., Batavia, H., Kaonga, N., Searle, S., Kwan, A., Goldberger, A., ... Ossman, J. (2010). Barriers and gaps affecting mHealth in low and middle income countries: Policy white paper. Washington, DC: Mhealth Alliance.

Nelson, L., Mulvaney, S., Gebretsadik, T., Johnson, K., \& Osborn, C. (2016). The Messaging for Diabetes (MED) intervention improves short-term medication adherence among low-income adults with type 2 diabetes. Journal of Behavioral Medicine, 39(6), 995-1000. doi: 10.1007/ s10865-016-9774-2

Nundy, S., Dick, J., Chou, C., Nocon, R., Chin, M., \& Peek, M. (2014). Mobile phone diabetes project led to improved glycemic control and net savings for Chicago Plan Participants. Health Affairs, 33(2), 265-272. doi: 10.1377/hlthaff.2013.0589

Organización Mundial de la Salud (2014). Global status report on noncommunicable diseases 2014. [online]. Disponible en: https://apps.who.int/iris/bitstream/hand le/10665/148114/9789241564854_eng.pdf;jsessionid=A BACEC4EE3B78598D74DA9318901AD95? sequence $=1$ [Acceso 11 Enero 2019].

Organización Mundial de la Salud (2016). mSalud: uso de las tecnologías móviles inalámbricas en la salud pública. [online]. Disponible en: http://apps.who.int/gb/ ebwha/pdf_files/EB139/B139_8-sp.pdf [Acceso 2 Enero 2019].

Organización Mundial de la Salud. (2016). Informe Mundial Sobre la diabetes. [online]. Disponible en: https://apps.who.int/iris/bitstream/handle/10665/204877/ WHO_NMH_NVI_16.3_spa.pdf?sequence=1 [Acceso 28 Diciembre 2019].

Porth, C., Gaspard, K., \& Noble, K. (2011). Essentials of pathophysiology: Concepts of Altered Health States (3rd ed.). Philadelphia: Wolters Kluwe/Lippincott Williams \& Wilkins.

Ryu, S. (2012). Book Review: mHealth: New horizons for health through mobile technologies: based on the findings of the Second Global Survey on eHealth (Global Observatory for eHealth Series, Volume 3). Healthcare Informatics Research, 18(3), 231.

Saffari, M., Ghanizadeh, G., \& Koenig, H. (2014). Health education via mobile text messaging for glycemic control in adults with type 2 diabetes: A systematic review and meta-analysis. Primary Care Diabetes, 8(4), 275-285. doi: 10.1016/j.pcd.2014.03.004

Thiessen, A. (2017). Educating Nursing students about patient SelfManagement Preventive Care to minimize Type II Diabetes Mellitus complications.

Wargny, M., Kleinebreil, L., Diop, S., Ndour-Mbaye, M., Ba, M., Balkau, B., \& Simon, D. (2018). SMSbased intervention in type 2 diabetes: clinical trial in Senegal. BMJ Innovations, 4(3), 142-146. doi: 10.1136/ bmjinnov-2018-000278

Wu, Y., Ding, Y., Tanaka, Y., \& Zhang, W. (2014). Risk factors contributing to Type 2 Diabetes and recent advances in the treatment and prevention. International Journal of Medical Sciences, 11(11), 1185-1200. 